\title{
QUÉ Y CUÁNTO APRENDEN DE MATEMÁTICAS LOS ESTUDIANTES DE BÁSICA CON EL MÉTODO SINGAPUR: EVALUACIÓN DE IMPACTO Y DE FACTORES INCIDENTES EN EL APRENDIZAJE, ENFATIZANDO EN LA BRECHA DE GÉNERO ${ }^{1}$
}

\author{
Lorena Espinoza \\ Claudia Matus \\ Joaquim Barbe \\ Jennypher Fuentes \\ Felipe Márquez ${ }^{2}$
}

\begin{abstract}
RESUMEN
Una problemática recurrente en la enseñanza de las matemáticas son los bajos porcentajes de logro que obtienen los estudiantes chilenos en mediciones nacionales e internacionales. Ello se vincula, en parte, con metodologías tradicionales de enseñanza más centradas en la apropiación de procedimientos muy específicos que en el desarrollo de una comprensión matemática en sus diversos aspectos y sentidos. Además, las mediciones dan cuenta de una importante brecha de género en esta área. Considerando la necesidad de revertir esta situación, el equipo proponente viene desarrollando un programa de asesoría a escuelas en el Método Singapur-Matemática. Esta experiencia permitió recoger la evidencia necesaria para realizar una investigación que aportara información acerca de su potencial efectividad. Los resultados muestran un impacto positivo en los aprendizajes de los estudiantes de las escuelas que utilizan esta metodología y una reducción significativa en la brecha de género. Asimismo, los textos escolares del método poseen un alto grado de cobertura, consistencia y coherencia con las bases curriculares. La capacitación docente y una organización institucional alineada con las demandas del método son factores relevantes que favorecen su apropiación. La tensión que supone el cambio de paradigma en la enseñanza es uno de los factores que más la dificultan.
\end{abstract}

Palabras clave: educación matemática, Método Singapur, prácticas docentes, resultados de aprendizaje.

1 Proyecto apoyado financieramente por CNED/ Convocatoria 2015.

2 Todas(os) las(os) investigadoras(es) se encuentran afiliados al Centro Félix Klein, Universidad de Santiago de Chile, Santiago, Chile. Contacto: lorena.espinoza@usach.cl 


\title{
WHAT AND HOW MUCH MATH ELEMENTARY SCHOOL STUDENTS LEARN, WITH THE SINGAPORE METHOD: EVALUATION OF IMPACT AND INCIDENT FACTORS ON LEARNING, WITH AN EMPHASIS ON THE GENDER GAP
}

\begin{abstract}
One of the most prevalent issues in math education in Chile is low achievement on national and international evaluations. It is partially explained by the traditional teaching methods, which are focused on very specific procedures instead of developing mathematical reasoning in all of its diverse aspects and meanings. Moreover, the test results show a significant gender gap in this area. Considering the need to correct this situation, this team, from The University of Santiago, is developing a consulting program for schools on the Singapore math method. This experience allowed the gathering of enough evidence to conduct a study that may provide information on its potential effectiveness. The results show a positive impact on student learning, along with significantly shrinking the gender gap, at the schools which used this method. Similarly, the method's textbooks are consistent and coherent with the national curriculum, covering most of the material. Teachers' capacity and an institutional organization aligned with the method's demands are relevant factors that favor its' appropriation. The resistance to change in the teaching paradigm presents one of the greatest obstacles.
\end{abstract}

Keywords: mathematical education, outcomes, Singapore Method, teaching practices.

\section{Antecedentes y formulación del problema}

Es sabido que el país requiere de estudios concretos acerca de experiencias exitosas de enseñanza de las matemáticas que puedan servir de referencia para levantar orientaciones de cómo llevarla a cabo de manera equitativa y de calidad. Por ello, esta investigación pretende responder cómo y en qué medida el Método Singapur contribuye a que niños y niñas logren aprendizajes matemáticos de calidad y desarrollen competencias en esta disciplina, al mismo tiempo que busca comprender cómo aporta a que los profesores gestionen una enseñanza en el aula acorde con las expectativas del currículo nacional. Por supuesto, la intención no es proponer el uso generalizado del método, sino más bien contar con resultados respecto de cómo apoyar a los docentes y a las escuelas para que logren elevar la calidad de la enseñanza de las matemáticas, comprendiendo cuáles son los factores que lo facilitan y cuáles lo dificultan, especialmente los referidos a la perspectiva de género. 
92 QUÉ Y CUÁNTO APRENDEN DE MATEMÁTICAS LOS ESTUDIANTES DE BÁSICA CON EL MÉTODO SINGAPUR: EVALUACIÓN DE IMPACTO Y DE FACTORES INCIDENTES EN EL APRENDIZAJE, ENFATIZANDO EN LA BRECHA DE GÉNERO - L. Espinoza, C. Matus, J. Barbe, J. Fuentes y F. Márquez

Esta investigación se centra en resultados de aprendizaje. En estudios posteriores se espera profundizar en otros aspectos involucrados con el logro de los aprendizajes, tales como prácticas docentes y tensiones frente al cambio. Así, las preguntas que guían la presente investigación son las siguientes:

- ¿El Método Singapur cubre todos los objetivos de aprendizaje del currículo de educación básica y es coherente con sus principios orientadores?

- ¿El Método Singapur promueve mejores resultados de aprendizaje matemático en estudiantes de $4^{\circ}$ básico que otras metodologías tradicionales de enseñanza?

- ¿El Método Singapur ofrece mayores oportunidades de aprendizaje matemático, facilitando la reducción de la brecha de género existente en el país?

- ¿Los profesores que utilizan el Método Singapur para realizar la enseñanza de las matemáticas demuestran una mayor variedad y riqueza de recursos y conocimientos didácticos que los profesores que no lo usan?

- ¿Las escuelas que utilizan el Método Singapur y que cuentan con una buena capacidad de organización, adecuadas condiciones laborales y con mayor satisfacción docente, favorecen los resultados de aprendizaje matemático de sus estudiantes?

\section{Contexto}

\section{1. ¿Qué es el Método Singapur?}

Según el Ministerio de Educación de Singapur (2012), el propósito general del currículo de matemáticas es asegurar que todos los estudiantes alcancen un nivel de dominio que les servirá para la vida, por lo que los objetivos generales de la educación matemática en Singapur están enfocados en posibilitar que los estudiantes:

- adquieran y apliquen conceptos y habilidades matemáticas;

- desarrollen habilidades cognitivas y metacognitivas, a través del enfoque de resolución de problemas matemáticos; y

- desarrollen actitudes positivas hacia las matemáticas. 
El Método Singapur busca el logro de los objetivos anteriores a través de una estructura pentagonal que articula el desarrollo de conceptos, habilidades, procesos matemáticos, metacognición y actitudes necesarias para el aprendizaje, cuyo foco central es la resolución de problemas en contextos significativos. Se plantea una metodología de acercamiento que evoluciona desde el uso de material concreto a la representación pictórica del problema y, posteriormente, a la utilización de símbolos y de un lenguaje más abstracto. A partir de este proceso, se espera que los estudiantes puedan reconocer la relación entre los datos y la incógnita del problema, comprenderlo mejor y resolverlo.

La propuesta está estructurada con criterios didácticos que relacionan las nociones matemáticas, promueven la apropiación progresiva de un lenguaje matemático y utilizan varios recursos como medios fundamentales para el aprendizaje. Los contenidos que los estudiantes deben y necesitan aprender en cada grado escolar se van desarrollando de manera gradual y sistemática para consolidarlos, articularlos y ampliarlos progresivamente, con un enfoque de aproximación en espiral, hasta lograr una construcción robusta del conocimiento. Así, los estudiantes son provistos de las experiencias de aprendizaje necesarias que, en su debido tiempo y en una secuencia pertinente, permiten el logro de los objetivos de aprendizaje.

\subsection{Método Singapur en Chile}

En Chile, el Centro Félix Klein (CFK) de investigación en Didáctica de la Matemática, perteneciente a la Universidad de Santiago, ha sido una de las instituciones responsables de transferir el Método Singapur al contexto nacional. Inicialmente este centro se encargó de estudiar, traducir y adaptar al contexto educacional chileno los textos My pals are here de Singapur. Así, produjo los primeros textos en español de esta metodología, titulados Pensar sin límites: Matemática Método Singapur.

En la actualidad el CFK colabora con la implementación del Método Singapur en más de 40 establecimientos educacionales del país. Esta incluye capacitación de docentes, jefes técnicos y 
94 QUÉ Y CUÁNTO APRENDEN DE MATEMÁTICAS LOS ESTUDIANTES DE BÁSICA CON EL MÉTODO SINGAPUR: EVALUACIÓN DE IMPACTO Y DE FACTORES INCIDENTES EN EL APRENDIZAJE, ENFATIZANDO EN LA BRECHA DE GÉNERO - L. Espinoza, C. Matus, J. Barbe, J. Fuentes y F. Márquez

directivos, observaciones y registro de clases, talleres de reflexión y profundización con los actores involucrados y también el diseño, construcción y análisis de los instrumentos de evaluación de aprendizajes en matemática de los estudiantes en los niveles que utilizan esta metodología. Al mismo tiempo, asesora al Ministerio de Educación Nacional de Colombia en un proyecto que busca capacitar a más de 2.700 docentes para que estos implementen el Método Singapur en sus escuelas.

Además de las escuelas asesoradas por el CFK, el Método Singapur se aplica en otros establecimientos educacionales del país, aunque en la práctica se evidencia que su nivel de implementación es variable: existen escuelas que solo utilizan los textos escolares adaptados de Singapur, mientras que otras, además del uso del texto, son apoyadas por instituciones que orientan su trabajo bajo distintos modelos de asesoría. Así, es relevante indagar cómo el Método Singapur contribuye a elevar los resultados de aprendizaje matemático de los estudiantes de una manera más efectiva.

\subsection{Evidencias de efectividad del Método Singapur}

Existe evidencia internacional y nacional que da cuenta de resultados de estudios del efecto de la metodología Singapur. Por ejemplo, a nivel internacional, el Instituto Americano de Investigación (AIR, por sus siglas en inglés) reportó la implementación de la metodología de cuatro experiencias piloto en Estados Unidos. En su informe, la AIR (2005) concluyó que dos de estas experiencias, las cuales tenían una población estable de estudiantes y un claro compromiso institucional para apoyar la introducción de los textos de Singapur, produjeron mejoras considerables en los resultados de los alumnos. En el primer caso, el porcentaje de estudiantes que alcanzó un nivel avanzado en $4^{\circ}$ grado, se incrementó en un 32\% en dos años. En el segundo caso, la proporción de estudiantes que puntuaron en el percentil 97 se incrementó en un 17\%. Por otra parte, en las otras dos experiencias los resultados fueron irregulares: en Montgomery County, donde el compromiso institucional con la implementación del método era más irregular, se observó una correlación positiva entre los resultados y el grado de entrenamiento profesional recibido por el cuerpo académico. 
Finalmente en Paterson, donde la rotación anual de estudiantes es de un $40 \%$, los resultados en $4^{\circ}$ grado no fueron mejores que los obtenidos dos años antes. El informe señala que estos establecimientos con alta rotación de estudiantes no son los más adecuados para evaluar los efectos acumulativos de la exposición a la metodología.

A su vez, el Educational Research Institute of America (ERIA) aplicó un estudio en Nueva Jersey. En el informe final, Conner (2010) señala que el uso del texto de Singapur permitió a los alumnos de $4^{\circ}$ grado quedar con 12,4 puntos por sobre la media en los resultados en la prueba estatal. A su vez, el grupo de comparación solo obtuvo 3,5 puntos más que el promedio. Además, se elevó en un 22\% el porcentaje de estudiantes en el nivel más alto, al contrario del grupo de comparación, el cual solo alcanzó un 4\%.

Algunos de los factores que influyen en la efectividad de la metodología Singapur en los contextos internacionales mencionados también se observan a nivel local. Por ejemplo, el Centro de Estudios del Mineduc (2013) señaló que aquellos profesores que recibieron capacitación directa por los singapurenses valoraron más la preparación, obtuvieron mejores resultados y manifestaron sentirse mucho más seguros a la hora de aplicar el método, mientras que quienes recibieron capacitación indirecta, tuvieron dificultades para utilizar los materiales y aplicar los contenidos. A partir de la valoración de los docentes hacia el método, el informe hace referencia a la necesidad de contar con apoyo institucional que incluya asesoría, monitoreo y supervisión de los encargados de la implementación del proyecto. Finalmente, el 90\% de los docentes percibió mayor interés y motivación en matemática por parte de los estudiantes, así como un mejor ambiente de aprendizaje, y una mayor seguridad a la hora de resolver problemas.

Respecto del estudio cuantitativo de impacto, el efecto del Método Singapur en el porcentaje de logro de los estudiantes de $2^{\circ}$ básico es de entre 2,02\% y 2,99\% mayor para quienes participaron por dos años en relación con los que no lo hicieron. Esta diferencia estadísticamente significativa evidenció un impacto positivo a corto plazo. 
96 QUÉ Y CUÁNTO APRENDEN DE MATEMÁTICAS LOS ESTUDIANTES DE BÁSICA CON EL MÉTODO SINGAPUR: EVALUACIÓN DE IMPACTO Y DE FACTORES INCIDENTES EN EL APRENDIZAJE, ENFATIZANDO EN LA BRECHA DE GÉNERO - L. Espinoza, C. Matus, J. Barbe, J. Fuentes y F. Márquez

En consecuencia, se puede concluir que el mejoramiento de los resultados en matemática mediante la implementación del Método Singapur no ocurre únicamente por adquirir los textos escolares basados en esta metodología. Es necesario considerar otros factores fundamentales para una implementación efectiva, como el grado de preparación de los docentes y el compromiso institucional para su adecuada implementación. Estos resultados son coherentes por lo propuesto en el reporte de McKinsey \& Company (2007) el cual sostiene que los sistemas educativos exitosos, entre los que se encuentra el de Singapur, resaltan la importancia de tres aspectos: conseguir a las personas más aptas para ejercer la docencia, desarrollarlas hasta convertirlas en instructores eficientes y garantizar que el sistema sea capaz de brindar la mejor instrucción posible a todos los niños.

\subsection{Formulación del problema}

A partir de los resultados en matemática obtenidos en evaluaciones estandarizadas tanto nacionales como internacionales en los últimos tiempos, se puede observar que se presentan dos problemas significativos en la educación chilena: el bajo nivel de logro de los estudiantes respecto de los estándares internacionales y la brecha existente en los resultados en matemática entre estudiantes hombres y mujeres.

\subsubsection{Desempeño de estudiantes chilenos en matemática}

Los resultados de las pruebas como PISA y TIMSS muestran que los logros de la mayoría de los alumnos en matemática son bajos considerando estos referentes internacionales. Por ejemplo, en la prueba TIMSS del año 2011 para el caso de matemática en $4^{\circ}$ básico, Chile obtuvo un puntaje promedio de 462 , ocupando el lugar 37 de entre 53 países participantes. El puntaje obtenido corresponde a 38 puntos por debajo del centro de la escala (Mullis, Martin, Foy y Arora, 2012). En esta misma prueba, en relación con los resultados obtenidos por los estudiantes nacionales según niveles de logro, Mullis et al. (2012) informan que un alto porcentaje de estudiantes se encuentra en el nivel más bajo y fuera de nivel, llegando a un 56\%. Por el contrario, el porcentaje de estudiantes ubicados en los niveles 
altos y avanzados fue de apenas el 12\%. En el caso de la prueba PISA del año 2012 los resultados también son desalentadores: según datos de la Organización para la Cooperación y el Desarrollo Económicos, OCDE (2014), Chile obtuvo 423 puntos, que corresponde a 71 puntos por debajo del promedio internacional, ubicándose en el lugar 50 de los 65 países evaluados. Además, el 52\% de los estudiantes chilenos se encuentra por debajo del nivel más básico, el 25\% está en el nivel mínimo y tan solo el $2 \%$ se encuentra en alguno de los dos niveles más altos.

En conclusión, a partir de los resultados en las evaluaciones TIMSS y PISA presentados anteriormente, se puede señalar que en ambas pruebas se destacan dos aspectos preocupantes: el bajo promedio de los resultados en matemática que obtiene Chile, y la distribución de los estudiantes entre los distintos niveles de logro de ambas pruebas. En efecto, se evidencia un predominio en los niveles más bajos, y muy pocos estudiantes que alcanzan un nivel de logro que cumpla los estándares internacionales.

Estos bajos resultados tienen una estrecha vinculación con insuficiencias en las prácticas de la enseñanza (Espinoza, Barbé y Gálvez, 2009) ya que, sobre la base de correlaciones entre desempeño docente y resultados de aprendizaje, se ha establecido que el factor más decisivo para mejorar la calidad de la educación es la efectividad de los profesores (OCDE, 2009).

\subsubsection{Desempeño por género}

La otra problemática que será abordada en esta investigación corresponde al comportamiento de los resultados en matemática según género. Respecto de este punto, la Organización de las Naciones Unidas para la Educación, la Ciencia y la Cultura, Unesco (2012) señala que en países de todo el mundo existen abundantes pruebas que apuntan a diferencias entre el rendimiento escolar de hombres y mujeres. Específicamente, las mujeres tienden a obtener mejores resultados en lectura, mientras que a los varones les suele ir mejor en matemática y ciencias. En nuestro país, este fenómeno se observa claramente en pruebas estandarizadas nacionales e internacionales: en 
98 QUÉ Y CUÁNTO APRENDEN DE MATEMÁTICAS LOS ESTUDIANTES DE BÁSICA CON EL MÉTODO SINGAPUR: EVALUACIÓN DE IMPACTO Y DE FACTORES INCIDENTES EN EL APRENDIZAJE, ENFATIZANDO EN LA BRECHA DE GÉNERO - L. Espinoza, C. Matus, J. Barbe, J. Fuentes y F. Márquez

el informe con los resultados de la prueba TIMSS del año 2011, Mullis et al. (2012) concluyen que en el caso de matemática en $4^{\circ}$ básico, la diferencia entre los puntajes de hombres y mujeres chilenas es de 9 puntos, la que se considera estadísticamente significativa a favor de los hombres; sin embargo, el promedio internacional en esta misma prueba muestra que no hay una diferencia significativa en los puntajes según género. Asimismo, el informe con resultados de la prueba PISA 2012 en matemáticas, muestra que Chile presenta la mayor brecha de género entre los países de la OCDE en educación media (OCDE, 2014). A su vez, la Agencia de Calidad de la Educación, ACE (2013) señala que los resultados de la prueba Simce han evidenciado hace varios años que existen diferencias en los puntajes según género en favor de los hombres.

En conclusión, considerando las problemáticas descritas anteriormente, existe una clara necesidad y también una oportunidad para que se realice una investigación sólida respecto de si el Método Singapur contribuye a elevar los resultados de aprendizaje matemático y disminuir la brecha de género. Por este motivo, resulta relevante investigar en torno al alcance del método a largo plazo, para orientar estrategias de enseñanza y políticas públicas en beneficio del aprendizaje matemático de los estudiantes.

\subsection{Objetivo general y objetivos específicos}

Como objetivo general se plantea evaluar cualitativa y cuantitativamente el impacto del uso de los textos Pensar sin límites -basados en la propuesta del Método Singapur-Matemática bajo la asesoría del Centro Félix Klein- en los resultados de aprendizaje matemático y en el desarrollo de habilidades y competencias de estudiantes de educación básica, identificando factores que favorecen la obtención de logros, así como aquellos que los dificultan a nivel de escuela, de docentes y de estudiantes, en especial analizando las brechas de género.

Por su parte, los objetivos específicos son los siguientes:

- Analizar la coherencia de la propuesta metodológica de los textos Pensar sin límites del Método Singapur-Matemática con las bases curriculares de educación básica de este subsector de 
aprendizaje, así como la cobertura de dichos textos en relación con los respectivos programas de estudio.

- Contrastar, mediante pruebas, los resultados de aprendizaje matemático que logran los estudiantes al utilizar sistemáticamente los libros de texto basados en el Método Singapur, con los aprendizajes que logran aquellos que utilizan otros libros de texto, en particular los entregados por el Ministerio de Educación a las escuelas públicas del país.

- Identificar factores que propician, y también que dificultan, la adquisición de habilidades y competencias matemáticas, analizando si son los mismos o distintos según género.

- Caracterizar los aprendizajes matemáticos que logran los estudiantes de educación básica, así como las competencias y habilidades matemáticas que desarrollan utilizando los libros de texto del Método Singapur, analizando si existen diferencias por género.

\section{Marco teórico}

La investigación se enmarca en la corriente del enfoque epistemológico en didáctica de las matemáticas que se construye a partir de teoría de situaciones de Guy Brousseau, y de los aportes de Yves Chevallard (transposición didáctica; teoría antropológica de lo didáctico), Michèle Artigue (reproductibilidad de situaciones), Régine Douady (teoría herramienta-objeto) y otros autores. Este enfoque constituye un marco teórico legitimado, que dispone de resultados sólidamente probados y es seguido por una parte importante de la comunidad científica internacional que investiga en esta área.

La evidencia teórica y empírica recogida ha puesto de manifiesto el papel decisivo que juegan los conocimientos disciplinarios específicos en la manera cómo enseñan los docentes, cómo aprenden los estudiantes y cómo se pueden modificar estas prácticas (Barquero, Bosch \& Gascón, 2013; Brousseau, 1997; Chevallard, 2012). De ahí también su relevancia para poder comprender mejor las prácticas docentes en este ámbito (Chevallard, 2012; Cochran-Smith \& Fries, 2005; Espinoza, Barbé y Gálvez 2011). 
100 QUÉ Y CUÁNTO APRENDEN DE MATEMÁTICAS LOS ESTUDIANTES DE BÁSICA CON EL MÉTODO SINGAPUR: EVALUACIÓN DE IMPACTO Y DE FACTORES INCIDENTES EN EL APRENDIZAJE, ENFATIZANDO EN LA BRECHA DE GÉNERO - L. Espinoza, C. Matus, J. Barbe, J. Fuentes y F. Márquez

La didáctica de las matemáticas estudia las interacciones y fenómenos que se producen entre el profesor y alumnos, a propósito de la transmisión de un saber matemático. Considera al sistema didáctico formado por tres polos: el profesor, el alumno y el saber. Puesto que se trata de un proyecto social de aprendizaje, la acción de enseñar es intencionada y está sujeta a las opciones culturales de la institución escolar. Lo que define al alumno y al profesor como tales es el proyecto de pasar de un estado inicial a uno final en relación con el saber. Las posiciones del alumno y el profesor son asimétricas en el sistema didáctico; en su estado inicial el profesor mantiene una relación privilegiada con el saber, en tanto que el alumno, si bien sostiene una relación con el saber antes de la enseñanza, esta es poco adecuada. En el estado final, el profesor desaparece y el alumno mantiene autónomamente una relación adecuada con el saber. Por ello, Brousseau (1994) afirma que el sistema didáctico contiene, desde el principio, el proyecto de su propia extinción.

El enfoque considera a la didáctica de las matemáticas como la "ciencia de las condiciones de creación y difusión de los conocimientos matemáticos útiles a los hombres y a sus instituciones" (Brousseau, 1994). Así, la investigación de cualquier problemática didáctica debe incorporar el análisis de los conocimientos matemáticos tal cual son reconstruidos en las instituciones de enseñanza, y su correspondiente proceso de transposición didáctica (Chevallard, 1985). Dicho proceso plantea la necesidad de ejercer una vigilancia epistemológica acerca de la distancia entre el saber matemático y el saber efectivamente enseñado. Estos análisis requieren de un modelo epistemológico del conocimiento matemático que sirva como referencia.

La vía desde la cual se aborda este problema de investigación utiliza con mayor énfasis la teoría antropológica de lo didáctico (Chevallard, 1999). Esta teoría adopta un punto de vista institucional de la problemática, situándola dentro del marco más general de las prácticas humanas. De acuerdo con dicho autor, la noción de organización matemática es propuesta para modelizar el conocimiento de esta disciplina. Toda organización matemática está compuesta por cuatro categorías de elementos: tipos de problemas, técnicas, tecnología y teoría. Chevallard postula que la teoría matemática 
emerge a propósito del estudio de una cuestión problemática que puede ser de naturaleza extra o intramatemática. Para estudiarla, se producen gestos matemáticos o técnicas que requerirán, en algún momento de su producción, un discurso tecnológico que permita hacerlos inteligibles, explicarlos y justificarlos en un primer nivel de reflexión. La teoría aparece, o se formaliza, cuando es necesaria una justificación y argumentación más rigurosa y consistente.

Las organizaciones matemáticas se construyen a través de procesos de creación y recreación de conocimientos. Así, la enseñanza y el aprendizaje de las matemáticas constituyen un proyecto común que esta teoría denomina "estudio de las matemáticas". La teoría de los momentos didácticos (que hace referencia al aspecto dinámico de la actividad matemática) propone, para modelizar dicho proyecto, seis momentos: del primer encuentro, exploratorio, del trabajo de la técnica, tecnológico-teórico, de la institucionalización y de la evaluación.

En la investigación se utilizaron las nociones de organización matemática y momentos didácticos para analizar los libros de texto del Método Singapur. Dicho análisis permitió establecer el grado de cobertura que estos alcanzan de las bases curriculares y programas de estudio, así como el grado de coherencia con las orientaciones didácticas. Las organizaciones matemáticas y didácticas resultan de calidad al ser completas, coherentes y articuladas dentro de un proyecto.

Cada organización matemática es el resultado de un proceso de estudio realizado en el seno de una institución determinada. Dicha institución impone restricciones para la realización del estudio; una estructura particular y unas condiciones de realización particulares. Para comprender los fenómenos de la enseñanza de las matemáticas abordados en esta investigación, en particular los relativos a logros en aprendizaje y las diferencias según género, será necesario completar los análisis, abordando las organizaciones didácticas que son construidas por profesores y estudiantes al realizar el estudio de las organizaciones matemáticas propuestas en los programas de estudio y textos escolares. Este análisis se realiza mediante las nociones de 
102 QUÉ Y CUÁNTO APRENDEN DE MATEMÁTICAS LOS ESTUDIANTES DE BÁSICA CON EL MÉTODO SINGAPUR: EVALUACIÓN DE IMPACTO Y DE FACTORES INCIDENTES EN EL APRENDIZAJE, ENFATIZANDO EN LA BRECHA DE GÉNERO - L. Espinoza, C. Matus, J. Barbe, J. Fuentes y F. Márquez

praxeología didáctica del profesor y praxeología didáctica del alumno, teoría de los momentos didácticos y contrato didáctico.

La noción de praxeología didáctica del profesor permite describir y analizar su actividad en términos de su componente práctica (el "saber-hacer" o praxis) y su componente teórica (el "saber" o logos). Se pueden distinguir, a su vez, dos niveles de análisis de dicha práctica. El primero se centra en los tipos de tareas y técnicas didácticas que utiliza el profesor para planificar, gestionar y evaluar el proceso de estudio de una organización matemática específica. Al lado de las técnicas didácticas se sitúan, en un segundo nivel, las tecnologías y teorías didácticas que permiten al profesor describir, explicar y justificar lo que hace y por qué lo hace. Generalmente estos elementos tecnológicos son difíciles de establecer, porque la actividad del profesor suele ser una práctica espontánea y poco reflexionada en ámbitos más específicos del conocimiento. Análogamente, la noción de praxeología didáctica del alumno permite describir su actividad en términos de su componente práctica y teórica. Se utilizaron estas nociones para analizar las clases observadas.

Dado el carácter institucional del proyecto educativo, se constata que el contrato didáctico (Brousseau, 1990) está fuertemente influenciado por restricciones que le impone la institución de enseñanza. La práctica del profesor, lejos de ser una actividad "autónoma", se encuentra sometida a todo tipo de imposiciones institucionales y culturales que gobiernan los aspectos generales, no específicos del contenido matemático, que afectan el entorno del estudio y que constituyen el contrato escolar (Chevallard, 1997).

Todas estas conceptualizaciones serán utilizadas en la investigación para identificar factores que favorecen y que dificultan los resultados en aprendizaje matemático. Dada la importante asimetría en Chile en los resultados de aprendizaje matemáticos al considerarlos por género (OCDE, 2014), especial interés tendrá esta variable en los análisis. 


\section{Marco metodológico}

\subsection{Metodología}

Esta investigación consta de tres partes: un estudio documental de los libros de texto Pensar sin límites basados en el Método Singapur, un estudio cuantitativo de impacto en los aprendizajes y desarrollo de habilidades de estudiantes de $4^{\circ}$ básico y un estudio cualitativo con el fin de caracterizar aspectos que han favorecido y dificultado la apropiación y la implementación de Método Singapur en escuelas que han implementado durante al menos seis años.

El estudio documental de los textos Pensar sin limites tuvo como propósito concluir cuál era la coherencia y cobertura de dichos textos respecto de los contenidos y las habilidades matemáticas establecidas en las bases curriculares 2012 y los correspondientes programas de estudio. Si bien es conocido por el sistema educativo en su conjunto que tales grados de cobertura y coherencia de los textos Pensar sin límites son elevados, se hace necesario describir, precisar y documentar dichos grados de relación y armonía curricular para argumentar y acreditar que el Método Singapur es una propuesta para la enseñanza de las matemáticas útil y potencialmente eficaz para profesores, estudiantes y escuelas de nuestro país. Este estudio documental tuvo tres etapas:

- Establecer el grado de cobertura de los textos Pensar sin límites respecto de las bases curriculares y programas, que consistió en determinar qué y cuántos elementos de las organizaciones matemáticas presentes en las bases curriculares están tratados en dichos textos. También se determinaron aquellos elementos de las organizaciones matemáticas identificadas en los textos Pensar sin límites que no aparecen explícitos en las organizaciones matemáticas de las bases curriculares, esto es, aquellos elementos de los textos que van más allá de estas, así como aquellos elementos que eventualmente podrían no estar tratados en Pensar sin limites.

- Establecer el grado de consistencia entre la progresión de los textos Pensar sin límites respecto de las bases curriculares y programas de estudio. Esto consistió en identificar el nivel o niveles en 
104 QUÉ Y CUÁNTO APRENDEN DE MATEMÁTICAS LOS ESTUDIANTES DE BÁSICA CON EL MÉTODO SINGAPUR: EVALUACIÓN DE IMPACTO Y DE FACTORES INCIDENTES EN EL APRENDIZAJE, ENFATIZANDO EN LA BRECHA DE GÉNERO - L. Espinoza, C. Matus, J. Barbe, J. Fuentes y F. Márquez

que aparecen tratados los elementos de las organizaciones matemáticas en dichas bases y programas, y contrastarlos con el o los niveles en que dichos elementos de organización matemática aparecen tratados en los textos Pensar sin límites. Un alto grado de consistencia en términos de la progresión da cuenta de que la trayectoria de aprendizaje matemático o recorrido propuesto a través de los distintos temas y por los diferentes cursos de educación básica es muy similar entre las bases curriculares y los textos Pensar sin límites.

- Establecer el grado de coherencia entre las bases curriculares y los textos Pensar sin límites, que consistió en identificar los principios fundamentales en torno a la enseñanza y el aprendizaje de las matemáticas que están en la base de las propuestas de ambos recursos curriculares, y se contrastaron para establecer similitudes y diferencias.

El estudio cuantitativo, por su parte, tuvo como fin contrastar el logro de los aprendizajes de los estudiantes que cursan $4^{\circ}$ básico con y sin la implementación de la metodología Singapur. Esto se realizó seleccionando una muestra de estudiantes que estaban trabajando con el Método Singapur en dicho nivel y una muestra comparable, pero que no utilizaba la metodología Singapur. Debido a las dificultades prácticas y éticas que implicó la conformación de grupos control no sometidos al método en las mismas escuelas participantes, se decidió realizar un diseño cuasiexperimental donde se seleccionó, mediante pareamiento o matching (Gertler, Martínez, Premand, Rawlings y Vermeersch, 2011), un grupo de comparación constituido por una muestra de estudiantes de $4^{\circ}$ básico de establecimientos que no utilizaban la metodología, y cuyas características son similares a las de los establecimientos que sí lo hacían. En otras palabras, cada escuela que implementaba el Método Singapur se asoció con otra cuya metodología de enseñanza de las matemáticas era otra. Para conformar las escuelas de comparación se consideraron las siguientes variables de control: comuna de origen, ruralidad, dependencia administrativa, nivel socioeconómico y puntaje Simce en matemática. A partir de los resguardos anteriores, se cauteló que estas variables no influyeran en las posibles diferencias en los resultados. Para recopilar la información 
de las escuelas se emplearon las bases de datos con las que cuenta la Agencia de Calidad de la Educación.

El estudio cualitativo tuvo como propósito comprender y describir aspectos clave de las transiciones que van experimentando los profesores de educación básica, los estudiantes y sus escuelas, al ir apropiándose de una nueva metodología de enseñanza de orientación constructivista como es el Método Singapur, bajo el apoyo de un programa de asesoría intensivo del CFK. Interesaba especialmente determinar los factores que favorecen la adquisición de habilidades y competencias matemáticas, así como aquellos que las dificultan. El análisis de las prácticas docentes, dimensión esencial de este estudio, se apoyó en una hipótesis acerca de la evolución de los sujetos en forma de "itinerarios de progresión" (Porlán, Rivero y Martín del Pozo, 1998). En este sentido, se pretendió identificar estadios de desarrollo de los docentes y de sus escuelas. Así, dado que este estudio requirió una comprensión amplia y profunda de cada dimensión considerada, esta parte de la investigación fue realizada a través de un estudio clínico de casos (Barbé, Bosch, Espinoza \& Gascón, 2005). Dado que fueron cinco las escuelas que han completado la enseñanza básica utilizando el Método Singapur desde kínder hasta $6^{\circ}$ básico bajo la asesoría del CFK, en este estudio de casos participaron tres de ellas.

Se levantó información en relación con las prácticas de los docentes que han implementado entre $4^{\circ}$ y $6^{\circ}$ básico entre los años 2014 y 2016. Bajo estas condiciones se realizaron 54 observaciones. Asimismo, se efectuaron tres grupos focales, uno en cada escuela, con todos los docentes de preescolar a $6^{\circ}$ básico y sus coordinadores técnicos.

Para describir los estadios en la apropiación del Método Singapur por parte de docentes, se utilizaron los criterios construidos por Gellert, Espinoza y Barbé (2013) para caracterizar procesos de estudios matemáticos que implementan una innovación pedagógica en la escuela.

Para describir el proceso de apropiación e instalación del método, así como identificar aspectos que lo favorecen y que lo 
106 QUÉ Y CUÁNTO APRENDEN DE MATEMÁTICAS LOS ESTUDIANTES DE BÁSICA CON EL MÉTODO SINGAPUR: EVALUACIÓN DE IMPACTO Y DE FACTORES INCIDENTES EN EL APRENDIZAJE, ENFATIZANDO EN LA BRECHA DE GÉNERO - L. Espinoza, C. Matus, J. Barbe, J. Fuentes y F. Márquez

dificultan, el estudio se apoyó en las distintas dimensiones que organizan el quehacer educativo de la escuela: a nivel de aula, de docentes, de directivos y comunidad educativa.

Para realizar este estudio fue necesario indagar en prácticas docentes, tecnología didáctica del docente, condiciones institucionales, trabajo en equipo docente y su cultura, percepciones de los docentes acerca de las valoraciones del Método Singapur por parte de padres y acerca de los cambios en las brechas de género en matemática.

\subsection{Población y muestra}

La evidencia nacional e internacional muestra que la efectividad de la metodología Singapur no se asegura únicamente con la implementación de los textos de estudio, sino que también inciden otros factores relevantes como el grado de preparación de los docentes, el compromiso institucional con la implementación del método y los años de ejecución (AIR, 2005; Conner, 2010). En consideración con lo anterior, es preciso señalar que las escuelas que forman parte de la población en estudio son establecimientos que no solo implementan la metodología con el uso de los textos de estudio, sino que forman parte de un proceso de asesoría integral que involucra, además del uso de los textos, lo siguiente:

- Once capacitaciones anuales a los docentes en relación con aspectos esenciales del método y estudio de los principales enfoques matemáticos y didácticos del texto.

- Ocho reuniones anuales con los equipos técnicos y directivos de las escuelas en los cuales se abordan temas relativos a la implementación de la metodología en el establecimiento.

- Entre cuatro y ocho visitas anuales a las escuelas en las que se realiza un acompañamiento a los docentes en aula y se los retroalimenta. En estas visitas también se sostienen reuniones con los equipos técnicos y/o directivos.

- Entre dos y cinco talleres adicionales entre los que se incluyen: talleres de inducción al Método Singapur para los docentes nuevos, taller de planificación de temáticas, talleres de evaluación y talleres de articulación curricular, entre otros. 
En el estudio cuantitativo se pretende medir el impacto del Método Singapur en los estudiantes de $4^{\circ}$ básico de los establecimientos que han implementado el Método Singapur bajo la asesoría del CFK y que utilizan los textos Pensar sin límites. En la actualidad el CFK colabora con la implementación en la metodología de aproximadamente 40 escuelas. De estas, hay 18 que implementan el Método Singapur en $4^{\circ}$ básico, por lo que los estudiantes de estas escuelas corresponden a la población en estudio.

De las 18 escuelas que implementan el Método Singapur en $4^{\circ}$ básico, 11 lograron asociarse con una escuela de comparación mediante la técnica de pareamiento o matching, considerando el control de todas las variables descritas en el apartado anterior. Finalmente, de las 11 escuelas de comparación posibles, solo seis de ellas accedieron a participar de la investigación. En conclusión, en este estudio se contó con una muestra de 12 escuelas, de las cuales seis corresponden a escuelas que implementan el Método Singapur en $4^{\circ}$ básico y seis que no lo hacen. Cabe destacar que la cantidad de escuelas que utilizan el Método Singapur utilizadas en la muestra corresponde a la tercera parte del total de escuelas que implementan en $4^{\circ}$ básico.

De las seis parejas de escuelas correspondientes a la muestra, cuatro pertenecen a la Región Metropolitana, una pareja es de la Región de Los Lagos y una de la Región de los Ríos. En cuanto a la dependencia administrativa, tres parejas de escuelas son particulares y las otras tres son particulares subvencionadas. Finalmente, en cuanto al nivel socioeconómico de las escuelas participantes, tres están catalogadas en un nivel alto y las otras tres, en un nivel medio. Respecto de la cantidad de estudiantes que participaron del estudio, se consideraron 680 estudiantes en total. De estos, 459 corresponden a estudiantes correspondientes al grupo de tratamiento y 221 son del grupo de comparación, no sometidos a la implementación del Método Singapur.

En el estudio cualitativo, la muestra quedó conformada por docentes, coordinadoras y directivos de tres escuelas que al momento de postular al fondo de investigación se encontraban implementando 
108 QUÉ Y CUÁNTO APRENDEN DE MATEMÁTICAS LOS ESTUDIANTES DE BÁSICA CON EL MÉTODO SINGAPUR: EVALUACIÓN DE IMPACTO Y DE FACTORES INCIDENTES EN EL APRENDIZAJE, ENFATIZANDO EN LA BRECHA DE GÉNERO - L. Espinoza, C. Matus, J. Barbe, J. Fuentes y F. Márquez

el Método Singapur desde educación parvularia hasta $6^{\circ}$ año básico. Dada la poca cantidad de escuelas que cumplen con esta condición, la selección fue realizada en forma intencionada, es decir, no aleatoria. De las escuelas participantes dos de ellas son de la Región Metropolitana y una es de la Región de Valparaíso.

\subsection{Instrumentos}

En el estudio documental se construyeron matrices de análisis que identifican y explican los elementos de las organizaciones matemáticas presentes en los textos Pensar sin limites y en las bases curriculares y programas de estudio, así como las habilidades y competencias matemáticas que propician y sus niveles de complejidad. Estas matrices organizan la información según nivel educativo. Dentro de cada nivel el análisis se organiza por ejes curriculares (números y operaciones; patrones y álgebra; medición; geometría; datos y azar) $y$, por temas dentro de cada eje, se identificaron elementos que parecen corresponder a las cuatro categorías que definen y componen una organización matemática, esto es: tareas, técnicas, tecnologías y teorías matemáticas, así como las habilidades matemáticas predominantemente movilizadas y su correspondiente nivel de complejidad. Posteriormente, y siguiendo la misma metodología, se analizaron los libros de textos Pensar sin límites por curso, para identificar los elementos de las organizaciones matemáticas presentes en ellos, por ejes del currículo y por temas dentro de los ejes, y las correspondientes habilidades matemáticas movilizadas.

En el estudio cuantitativo, se aplicó un postest tanto al grupo de tratamiento como al de comparación. Este postest consistió en una prueba que medía los conocimientos y habilidades matemáticas de los estudiantes de $4^{\circ}$ básico en matemática, según los objetivos de aprendizaje definidos en las bases curriculares. La validez de contenido del instrumento fue verificada por profesores de matemática y expertos en didáctica. Posteriormente, este instrumento fue aplicado durante el mes de diciembre de 2014 a estudiantes de $4^{\circ}$ básico y, a modo de pilotaje, se seleccionaron los resultados de 320 estudiantes de establecimientos con diferente nivel socioeconómico. En el análisis psicométrico del instrumento se obtuvo que la confiabilidad 
de los resultados del instrumento, determinada por el alfa de Cronbach, arroja un valor de 0,7870. Además se realizaron análisis de consistencia interna y validez de criterio, en el cual se obtuvo una alta correlación $(0,94)$ entre esta prueba y los puntajes Simce de matemática $4^{\circ}$ básico del año 2014. Finalmente, se realizaron análisis de ítems a partir de la teoría clásica de medición y la teoría de respuesta al ítem. En consecuencia, el instrumento final que se aplicó a los grupos de tratamiento y de comparación consistió en una prueba de 27 preguntas de opción múltiple con cuatro alternativas cada una, de las cuales solo una era la correcta.

En el estudio cualitativo se utilizaron varios instrumentos validados por el CFK, siguiendo los procedimientos estadísticos clásicos. Los instrumentos utilizados para cada aspecto de la investigación eran: pauta de observación de clases (análisis de prácticas docentes y estudiantes); registros de visitas a escuelas (factores institucionales); bitácoras (prácticas docentes); pruebas de conocimientos matemáticos y didácticos aplicadas a los docentes (tecnología didáctica de docentes); encuesta (percepciones de docentes). También se realizaron grupos focales con los equipos de docentes de educación parvularia y básica de las escuelas y sus coordinadores (tecnología didáctica de docentes y del equipo directivo).

\section{Análisis de los resultados y discusión}

A continuación se presentarán los principales resultados obtenidos en cada uno de los estudios realizados.

\subsection{Estudio documental}

El levantamiento de información y la construcción de las matrices de análisis siguiendo la metodología expuesta anteriormente permitió obtener los siguientes resultados.

\subsubsection{Grado de cobertura curricular}

El trabajo metodológico parte por construir matrices de análisis que identifican y describen los elementos de las organizaciones 
110 QUÉ Y CUÁNTO APRENDEN DE MATEMÁTICAS LOS ESTUDIANTES DE BÁSICA CON EL MÉTODO SINGAPUR: EVALUACIÓN DE IMPACTO Y DE FACTORES INCIDENTES EN EL APRENDIZAJE, ENFATIZANDO EN LA BRECHA DE GÉNERO - L. Espinoza, C. Matus, J. Barbe, J. Fuentes y F. Márquez

matemáticas presentes en las bases curriculares de $1^{\circ}$ a $6^{\circ}$ básico, así como las habilidades y competencias matemáticas que propician y sus niveles de complejidad. El estudio se realizó en función de los ejes curriculares (números y operaciones; medición; geometría; y estadística y probabilidad) y por temas dentro de cada eje, identificando los objetivos de aprendizaje de las bases curriculares que se relacionaban con dichos temas. Además, dentro de cada objetivo de aprendizaje se identificó cada una de las tareas matemáticas presentes. Siguiendo una metodología similar, se construyó una segunda matriz en la que se determinaron las tareas matemáticas presentes en los capítulos de los textos Pensar sin límites de cada nivel. Luego, con el fin de definir el grado de cobertura de dichos textos respecto de las bases curriculares, en una tercera matriz se buscó la correspondencia entre las tareas de las bases curriculares y las de Pensar sin límites, la que podría ser mayor o menor en la medida en que las tareas de las primeras estén en algún grado cubiertas por las segundas. Además, se indicaron las condiciones propiciadas en los textos Pensar sin límites que permitían responder a dichas tareas.

Más adelante, se construyó una cuarta matriz que resumía todas aquellas tareas matemáticas presentes en Pensar sin límites que no eran abordadas por las bases curriculares de $1^{\circ}$ a $6^{\circ}$. Esto motivó la revisión de dichas bases en los niveles de $7^{\circ}$ y $8^{\circ}$ básicos, logrando relacionar gran parte de esas tareas de los textos con algunos objetivos de aprendizaje de esos niveles.

Con el fin de ejemplificar el tipo de análisis hecho y de la matrices descritas, se muestra un fragmento de dos de las cuatro matrices construidas.

Tabla 1. Matriz de análisis de correspondencia entre bases curriculares y Pensar sin límites

\begin{tabular}{|l|l|l|l|l|}
\cline { 4 - 5 } & \multicolumn{2}{l|}{$\begin{array}{l}\text { Capítulo de Pensar } \\
\text { sin límites }\end{array}$} \\
\hline Eje & Tema & $\begin{array}{l}\text { Objetivo de } \\
\text { aprendizaje }\end{array}$ & Tareas & 10 básico \\
\hline $\begin{array}{l}\text { Números y } \\
\text { operaciones }\end{array}$ & SND & $\begin{array}{l}\text { Objetivo de } \\
\text { aprendizaje 3 } \\
2^{0} \text { básico }\end{array}$ & $\begin{array}{l}\text { Ordenar números del 0 al 100, } \\
\text { de menor a mayor y viceversa }\end{array}$ & Capítulo 17 \\
\cline { 4 - 5 } & Comparar números del 0 al 100 & Capítulo 17 \\
\hline
\end{tabular}


Tabla 2. Tareas de Pensar sin límites no abordadas en las bases curriculares

\begin{tabular}{l|l|l|l|l}
\hline Eje & Tema & Tareas & Nivel Pensar sin límites & $\begin{array}{l}\text { Niveles bases } \\
\text { curriculares }\end{array}$ \\
\hline \multirow{2}{*}{$\begin{array}{l}\text { Números y } \\
\text { operaciones }\end{array}$} & SND & $\begin{array}{l}\text { Redondear para } \\
\text { estimar si la } \\
\text { respuesta a un } \\
\text { cálculo es razonable }\end{array}$ & $3^{0}-4^{0}$ & Ninguno \\
\cline { 2 - 5 } & $\begin{array}{l}\text { Multiplicar } \\
\text { fracciones propias }\end{array}$ & $6^{0}$ & 70 \\
\hline
\end{tabular}

Al contrastar las matrices resultantes del análisis de las bases curriculares y los textos Pensar sin limites, se observa muy claramente que dichos libros abordan prácticamente la totalidad de los objetivos de aprendizaje de las bases curriculares y de los programas de estudio, y estimulan el desarrollo de las mismas habilidades matemáticas. Las organizaciones matemáticas de ambos recursos curriculares comparten elementos estructurantes que son similares. No obstante, hay que señalar que existen algunos temas, y por tanto algunas organizaciones matemáticas, que las bases curriculares proponen para ser estudiadas en $7^{\circ}$ y/o en $8^{\circ}$ básico, que en los textos Pensar sin límites son tratadas en cursos anteriores a estos, como por ejemplo, el caso de la multiplicación de fracciones que en Pensar sin límites se estudia en $6^{\circ}$ básico, mientras que en las bases curriculares se propone en $7^{\circ}$ básico. Esto señala que Pensar sin límites cubre la totalidad de los objetivos de aprendizaje de las bases curriculares, e incluso incorpora algunas temáticas no propuestas en las mismas, tal es el caso del cálculo mental, donde Pensar sin límites agrega la estimación como un medio para analizar si los resultados obtenidos son o no razonables. Un análisis superficial de la cobertura podría hacer pensar que el estudio de las propiedades de los números no son tratadas en los textos Pensar sin límites, lo cual dista de la realidad y un ejemplo claro es cómo se propone utilizar la distributividad en la multiplicación para construir las tablas de multiplicar. Otra conclusión errada sería pensar que en Pensar sin límites el tratamiento del álgebra es reducido o nulo, lo que se puede atribuir a que en dichos textos de $1^{\circ}$ a $4^{\circ}$ básico, el eje de álgebra se trata de forma integrada a los otros ejes, impidiendo a los docentes visualizar este eje claramente. Acerca de las habilidades, es posible afirmar que en Pensar sin límites se propicia el desarrollo de las mismas habilidades que en las bases curriculares, 
112 QUÉ Y CUÁNTO APRENDEN DE MATEMÁTICAS LOS ESTUDIANTES DE BÁSICA CON EL MÉTODO SINGAPUR: EVALUACIÓN DE IMPACTO Y DE FACTORES INCIDENTES EN EL APRENDIZAJE, ENFATIZANDO EN LA BRECHA DE GÉNERO - L. Espinoza, C. Matus, J. Barbe, J. Fuentes y F. Márquez

con ejemplos claros al referirse a la resolución de problemas presente en todos los niveles de enseñanza, al igual que el modelamiento, la representación y la argumentación y comunicación.

\subsubsection{Grado de consistencia}

Las trayectorias de aprendizaje o recorridos propuestos por los distintos ejes del currículo de matemáticas, por los temas dentro de los ejes a lo largo de los seis cursos de la enseñanza básica, son bastante similares entre las bases curriculares y los textos Pensar sin limites. Las principales diferencias radican en que el estudio de determinados tópicos de Pensar sin límites se adelanta uno o dos niveles de enseñanza respecto de las bases curriculares y, a su vez, es muy habitual encontrar que el estudio de ciertos tópicos nucleares de los textos Pensar sin límites se desglosan en varios niveles, a diferencia de las bases curriculares, donde es usual encontrar un tópico o tarea en un solo nivel, especialmente en los ejes de geometría y medición; ello obedece al principio del Método Singapur de un currículo en espiral. Así también, Pensar sin límites considera algunos temas matemáticos de manera transversal dentro de un mismo nivel, motivo por el cual la operatoria se introduce en los primeros capítulos del texto para luego ser aplicada en todos los demás, en particular en la resolución de problemas.

A su vez, del análisis se puede apreciar cómo en Pensar sin límites se retrasa el estudio de algunas tareas muy específicas de las bases curriculares. Ello, a nuestro parecer, con el propósito de disponer de determinados conocimientos y herramientas matemáticas, que bajo el enfoque del Método Singapur son esenciales para el estudio de dichas tareas. Un ejemplo claro es la decisión de introducir la recta numérica en los textos Pensar sin límites en $4^{\circ}$ básico, cuando ya se han estudiado las fracciones y los números mixtos, a diferencia de las bases curriculares que proponen su introducción en $3^{\circ}$ básico. Igual ocurre con el estudio de las ecuaciones y las inecuaciones, las que son abordadas por Pensar sin limites en $5^{\circ}$ básico, mientras que en las bases curriculares se proponen desde $3^{\circ}$ básico. 


\subsubsection{Grado de coherencia}

Al contrastar los principios fundamentales que están en la estructura de las bases curriculares y los textos Pensar sin limites, se observa un alto grado de coherencia. En algunos casos, los principios aparecen descritos o evocados de distintas maneras y con distinto lenguaje. Así por ejemplo, ahí donde las bases curriculares hablan de progresión en complejidad, los textos Pensar sin límites hablan de currículum en espiral. Asimismo, el principio del Método Singapur relacionado con el uso de material concreto y de distintas representaciones, es claramente descrito en las bases curriculares y de manera más sintetizada en el principio metodológico llamado COPISI (concreto-pictóricosimbólico). En este sentido, el uso de materiales concretos destaca en el Método Singapur, dado que se sugieren vinculados con los otros registros de representación desde $1^{\circ}$ a $6^{\circ}$ básico, como por ejemplo, los discos fraccionarios, la regla, entre otros, y si bien se evidencia una disminución del uso de los materiales concretos de $5^{\circ}$ básico en adelante, esto ocurre en aquellos casos en que el tema matemático ya ha sido trabajado en años anteriores con los materiales concretos y en los niveles superiores se busca priorizar lo pictórico y simbólico.

Los procesos metacognitivos explícitamente fomentados en los textos Pensar sin límites aparecen implícitos en el discurso declarado de las bases curriculares y los programas de estudio. Sin embargo, es posible reconocerlos como medios necesarios para desarrollar todas las habilidades matemáticas propiciadas por las bases curriculares, especialmente la de comunicar. Tanto en las bases curriculares como en Pensar sin límites se mencionan las oportunidades de aprendizaje matemático para todos los estudiantes, en busca de avanzar, ya sea individual o colectivamente, en su propio proceso de aprendizaje. El éxito de este principio común radica en la diversidad de actividades elegidas y los tipos de representaciones con los que cuenta el estudiante.

En consecuencia, dados los altos grados de cobertura de los textos Pensar sin límites en relación con las bases curriculares y programas de estudio que se han encontrado, el elevado grado de consistencia entre las progresiones de ambos recursos curriculares y el alto grado de coherencia entre sus principios fundamentales, se 
114 QUÉ Y CUÁNTO APRENDEN DE MATEMÁTICAS LOS ESTUDIANTES DE BÁSICA CON EL MÉTODO SINGAPUR: EVALUACIÓN DE IMPACTO Y DE FACTORES INCIDENTES EN EL APRENDIZAJE, ENFATIZANDO EN LA BRECHA DE GÉNERO - L. Espinoza, C. Matus, J. Barbe, J. Fuentes y F. Márquez

puede afirmar que los libros Pensar sin límites son una destacada y pertinente interpretación de las propuestas de aprendizaje realizadas en las bases curriculares y programas de estudio de educación básica en matemáticas. Además de poner los énfasis en los mismos aspectos del proceso de aprendizaje, los textos Pensar sin limites proponen estudios matemáticos a través de los distintos cursos de la educación básica y en sus distintos ejes curriculares, que resultan incluso más profundos y secuenciados que los apuntados en las bases curriculares y programas de estudio correspondientes. Esto, gracias a que los grados de consistencia entre las trayectorias de aprendizaje de ambos recursos curriculares mostraron que los textos Pensar sin límites proponen estudios matemáticos, en algunos temas del currículo, que resultan más dilatados en el tiempo que los que proponen las bases curriculares. Al mismo tiempo, las habilidades matemáticas están ampliamente tratadas en dichos textos, tal como mostró el estudio acerca de los grados de cobertura, incluso con un mayor desglose en algunos temas matemáticos de la trayectoria de aprendizaje del currículo nacional.

\subsection{Estudio cuantitativo}

En la Tabla 3 se muestran los porcentajes de logro promedios obtenidos por los estudiantes correspondientes a los grupos de tratamiento y de comparación a nivel general, y también según habilidad matemática.

Tabla 3. Porcentajes de logro de ambos grupos en general y según habilidad matemática

\begin{tabular}{l|l|c|c}
\hline & & $\begin{array}{c}\text { Grupo de } \\
\text { tratamiento } \\
N=459\end{array}$ & $\begin{array}{c}\text { Grupo de } \\
\text { comparación } \\
N=221\end{array}$ \\
\hline \multirow{2}{*}{ General } & \% de logro promedio & 77,68 & 72,92 \\
\cline { 2 - 4 } & Desviación estándar & 15,93 & 15,53 \\
\hline \multirow{2}{*}{$\begin{array}{l}\text { Hab. } \\
\text { Resolver problemas }\end{array}$} & \% de logro promedio & 78,69 & 75,11 \\
\cline { 2 - 4 } Hab. & Desviación estándar & 18,37 & 19,85 \\
\hline Manipular expresiones matemáticas & \% de logro promedio & 74,35 & 67,63 \\
\cline { 2 - 4 } & Desviación estándar & 18,65 & 17,10 \\
\hline Hab. & \% de logro promedio & 82,10 & 78,96 \\
\cline { 2 - 4 } Representar & Desviación estándar & 20,45 & 21,93 \\
\hline
\end{tabular}


De acuerdo con la información anterior, el porcentaje de logro promedio de los estudiantes que implementan la metodología es 4,76 puntos porcentuales mayor que el porcentaje de logro promedio de los alumnos de las escuelas de comparación. Para determinar la significancia estadística de esta diferencia, se realizó un T-Test para dos muestras independientes. Los resultados de esta prueba estadística permiten afirmar con un $95 \%$ de confianza que la diferencia entre los puntajes, a favor de los estudiantes de las escuelas que implementan el Método Singapur, es estadísticamente significativa.

En definitiva, los resultados del T-test arrojan que los estudiantes que implementan el Método Singapur obtuvieron en promedio mejores resultados que aquellos estudiantes que no lo implementan. Esto quiere decir que el Método Singapur es una buena opción para los estudiantes chilenos, puesto que estos logran mejores niveles de aprendizaje, en relación con las bases curriculares, que aquellos que estudian con otras metodologías.

El instrumento fue diseñado para poder evaluar, además de los conocimientos matemáticos, el nivel de desarrollo de habilidades matemáticas de los estudiantes, puesto que también son un eje central de las bases curriculares. Las habilidades matemáticas evaluadas en este instrumento fueron manipular expresiones matemáticas, resolver problemas y representar. La habilidad argumentar y comunicar no fue considerada, puesto que es no es pertinente evaluarla mediante preguntas de respuesta seleccionada. En cuanto a la habilidad de modelar, en estos niveles iniciales queda subsumida en la habilidad de resolver problemas, por lo que se evalúa implícitamente.

Al considerar los resultados según las habilidades matemáticas evaluadas en el instrumento, se evidencia que se obtuvieron resultados estadísticamente significativos a favor de los estudiantes que implementan el Método Singapur en las habilidades de resolver problemas y manipular expresiones matemáticas, con una diferencia de 3,58 y 6,72 puntos porcentuales respectivamente. En cambio, no hubo diferencias significativas en los porcentajes de logro promedio asociados a la habilidad de representar. 
116 QUÉ Y CUÁNTO APRENDEN DE MATEMÁTICAS LOS ESTUDIANTES DE BÁSICA CON EL MÉTODO SINGAPUR: EVALUACIÓN DE IMPACTO Y DE FACTORES INCIDENTES EN EL APRENDIZAJE, ENFATIZANDO EN LA BRECHA DE GÉNERO - L. Espinoza, C. Matus, J. Barbe, J. Fuentes y F. Márquez

Respecto de los resultados según género, la siguiente tabla resume los porcentajes de logro generales y según habilidad de los hombres y mujeres de ambos grupos:

Tabla 4. Porcentajes de logro según habilidad de ambos grupos y según género

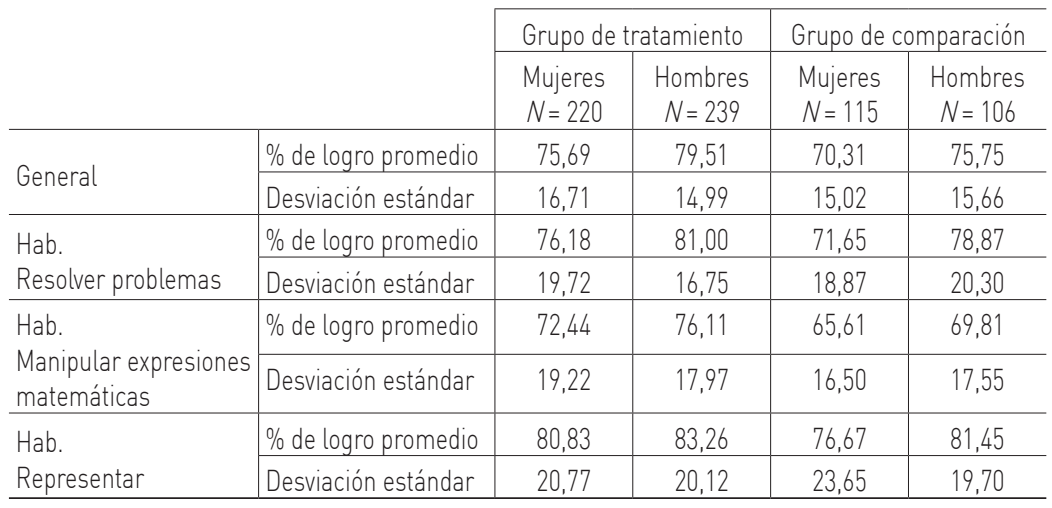

Respecto de la tabla anterior, se evidencia que en relación con los resultados obtenidos por las mujeres de ambos grupos, hay una diferencia de 5,38 puntos porcentuales a favor de aquellas que implementan el Método Singapur. Esta diferencia resulta ser estadísticamente significativa. En el caso de los hombres ocurre algo similar: hay una diferencia significativa de 3,76 puntos porcentuales a favor de los hombres que implementan Método Singapur. A su vez, si se consideran los resultados por habilidad matemática, se evidencia que las mujeres que implementan el Método Singapur obtienen resultados significativamente mejores que las que no implementan esta metodología en las habilidades de resolver problemas y manipular expresiones matemáticas, mientras que en el caso de la habilidad representar, no se observan diferencias estadísticamente significativas. A su vez, en el caso de los hombres, aquellos que implementan el Método Singapur obtuvieron porcentajes de logro significativamente más altos solo en la habilidad de manipular expresiones matemáticas. Respecto de las habilidades resolver problemas y representar no se evidenciaron diferencias estadísticamente significativas.

En relación con la brecha de género, se observa en ambos grupos diferencias de género que son estadísticamente significativas. En el caso del grupo que implementa el Método Singapur, la brecha de 
género alcanzó los 3,82 puntos porcentuales a favor de los hombres, mientras que en el grupo de comparación, esta fue de 5,44 puntos porcentuales también a favor de los hombres. Esta diferencia entre géneros es consistente con los resultados obtenidos en numerosos estudios internacionales, siendo atribuibles a un fuerte sesgo sociocultural respecto de la creencia de que las matemáticas son un dominio masculino. Sin embargo, en el grupo que implementa la metodología Singapur la brecha de género es significativamente menor que en el grupo de comparación, por lo que podemos asegurar que la implementación de la metodología Singapur influye positivamente en la disminución en la brecha de género en los resultados en matemática, a pesar de que aún queda trabajo por hacer, puesto que se siguen observando diferencias en los resultados de aprendizaje a favor de los hombres.

Respecto de la brecha de género en los resultados según habilidad matemática, se evidencia que tanto en el grupo de tratamiento como en el de comparación, los hombres presentan mejores resultados que las mujeres en la habilidad de resolver problemas. En el caso de la habilidad de manipular expresiones matemáticas, hubo diferencias a favor de los hombres solo en el caso del grupo de tratamiento, mientras que en el grupo de comparación no hubo diferencias estadísticamente significativas en los resultados entre hombres y mujeres. Por último, respecto de la habilidad de representar, no hubo diferencias de género estadísticamente significativas en ninguno de los dos grupos que rindieron la evaluación.

Finalmente, se observó que en la habilidad resolver problemas, la brecha de género fue estadísticamente inferior en el caso de los estudiantes que implementa el Método Singapur que aquellos del grupo de comparación (4,82 y 7,22 puntos porcentuales, respectivamente), lo que da cuenta de la eficacia del Método Singapur para reducir la brecha de género, puesto que dicha reducción se manifiesta en un aspecto medular del aprendizaje de las matemáticas como es la resolución de problemas. 
118 QUÉ Y CUÁNTO APRENDEN DE MATEMÁTICAS LOS ESTUDIANTES DE BÁSICA CON EL MÉTODO SINGAPUR: EVALUACIÓN DE IMPACTO Y DE FACTORES INCIDENTES EN EL APRENDIZAJE, ENFATIZANDO EN LA BRECHA DE GÉNERO - L. Espinoza, C. Matus, J. Barbe, J. Fuentes y F. Márquez

\subsection{Estudio cualitativo}

Este estudio indagó en torno a prácticas docentes en aula, tecnología didáctica de docentes, y condiciones institucionales para el trabajo pedagógico. A continuación se presentan los resultados organizados en tres apartados.

\subsubsection{Prácticas docentes en aula y tecnología didáctica de docentes} El análisis de las prácticas docentes en aula se realizó a través de una pauta de observación que permitía registrar los aspectos esenciales de la gestión de una clase, así como el grado en que estos aspectos se manifiestan en ella. La pauta contiene 13 indicadores que especifican aspectos esenciales de una clase de matemática según el enfoque teórico asumido. A su vez, cada indicador contempla tres descriptores que se corresponden con una graduación que cuantifica el nivel de logro de cada indicador. Esta graduación va de 1 a 3, donde 1 es el grado más bajo de presencia del indicador y 3 el más alto. Además, para cada indicador se contempla la categoría NA (No aplica), en aquellos casos que, por la naturaleza de la clase observada, ese indicador no podía estar presente.

Se observaron dos clases por cada profesor participante y se recuperaron las pautas de observación de los años anteriores al estudio (2014 y 2015) de la mayoría de estos docentes. El análisis se realizó primero por profesor y luego por escuela, considerando a todos los participantes.

Con la finalidad de centrar el análisis en los aspectos más representativos del Método Singapur, en la Tabla 5 se presenta, a modo de ejemplo, la frecuencia de tres de los 13 indicadores que resultan clave para el Método Singapur, y que corresponden a los docentes de las tres escuelas. En este ejemplo se ha omitido el descriptor NA puesto que se han considerado las observaciones de aquellas clases que, por su naturaleza, tendrían que haber cumplido en algún grado este indicador. 
Tabla 5. Frecuencia porcentual de algunos indicadores definidos en las pautas de observación

\begin{tabular}{l|c|c|c}
\hline Indicadores & 1 & 2 & 3 \\
\hline $\begin{array}{l}\text { 1. El profesor gestiona la actividad propuesta generando las } \\
\text { condiciones para que los alumnos exploren sobre un nuevo } \\
\text { conocimiento matemático en estudio. }\end{array}$ & $24 \%$ & $34 \%$ & $42 \%$ \\
\hline $\begin{array}{l}\text { 2. El profesor coordina los distintos registros de representación } \\
\text { (concreto - pictórico - simbólico) de la noción matemática en estudio. }\end{array}$ & $17 \%$ & $26 \%$ & $57 \%$ \\
\hline $\begin{array}{l}\text { 3. El profesor promueve que los estudiantes argumenten sus } \\
\text { respuestas y procedimientos. }\end{array}$ & $17 \%$ & $22 \%$ & $61 \%$ \\
\hline
\end{tabular}

De estos tres indicadores clave, y con el propósito de mostrar el tipo de análisis realizado de cada indicador, a continuación se presenta lo obtenido en uno de ellos.

En cuanto al indicador 1, según lo observado en las aulas de estas tres escuelas, en un 42\% de las clases observadas, la gestión de las actividades que plantean los docentes para la exploración de un nuevo conocimiento, tiende a promover una auténtica indagación por parte de los estudiantes (Nivel 3 del indicador). El profesor formula preguntas precisas para problematizar a los niños y son ellos quienes producen una respuesta para resolverla. En años anteriores, el porcentaje en el Nivel 3 fue de un 30\%, lo que estaría indicando una mejora evidente y generalizada en este indicador de carácter clave para el Método Singapur.

Asimismo, un 24\% de los profesores observados están en el Nivel 1 de este indicador. Esto implica que, aunque las características de las actividades permitirían una exploración genuina, los docentes entregan inmediatamente indicaciones explícitas para realizarlas, o bien, dichas indicaciones resultan insuficientes, por lo que no se logra que los estudiantes vivencien un proceso de exploración. El porcentaje de docentes en el Nivel 1 de años anteriores fue de un $31 \%$, lo que estaría indicando que en estas escuelas han disminuido las clases donde la exploración aparece obstaculizada.

A partir del análisis de las pautas de observación de clases de los docentes de las tres escuelas es posible afirmar que, de manera general, se observan importantes avances en la mayoría de los descriptores de la pauta que ahondan en aspectos clave de una enseñanza de las 
120 QUÉ Y CUÁNTO APRENDEN DE MATEMÁTICAS LOS ESTUDIANTES DE BÁSICA CON EL MÉTODO SINGAPUR: EVALUACIÓN DE IMPACTO Y DE FACTORES INCIDENTES EN EL APRENDIZAJE, ENFATIZANDO EN LA BRECHA DE GÉNERO - L. Espinoza, C. Matus, J. Barbe, J. Fuentes y F. Márquez

matemáticas en aula basada en el Método Singapur. Los docentes de estas escuelas, en general, han logrado comprender y gestionar en aula buenas problematizaciones y han abierto espacios importantes para la exploración auténtica de los estudiantes. Asimismo, han logrado que sus estudiantes desarrollen una actitud de cuestionamiento constante, se pregunten los porqué y para qué de los conocimientos, y reflexionen acerca de cómo ellos los han razonado y producido, propiciando de este modo razonamientos metacognitivos. Claro está que los mayores porcentajes de docentes no están ubicados en los más altos niveles de los indicadores, sin embargo, comparándolos en años diferentes, es posible verificar importantes avances.

En cuanto a la tecnología didáctica de los docentes, el estudio reveló cuestiones interesantes. En primer lugar, que la profundidad de los procesos reflexivos de los docentes no está estrictamente relacionada con años de experiencia. No hacen mejores reflexiones necesariamente los docentes que llevan más tiempo implementando el Método Singapur, aunque por supuesto la experiencia de implementación sostenida aparece como un factor que ofrece importantes oportunidades para lograr reflexiones y comprensiones profundas.

Hay aspectos de la tecnología didáctica relacionada con el Método Singapur que son comunes a la mayoría de los profesores entrevistados. Entre ellos, la importancia que le otorgan a que los estudiantes resuelvan problemas de distintas maneras, también que expliquen y argumenten sus respuestas. Este convencimiento dicen tenerlo, no solo a partir de lo que han estudiado acerca del Método Singapur, sino principalmente por la gran motivación que este método ha provocado en sus estudiantes. Valoran muy positivamente el nuevo lenguaje que instaló el Método Singapur en sus escuelas. Por otro lado, el cambio de paradigma que este método plantea les resulta muy difícil de gestionar en el aula ya que, frente a cualquier dificultad, la forma tradicional de enseñanza emerge de manera automática. Por ejemplo, al presentarse un error en la clase, la gestión de los profesores tiende a reducirse a explicar "la respuesta" a sus estudiantes e instalar el conocimiento a modo de receta. 
Así como se hallaron aspectos comunes, también aparecieron marcadas diferencias entre la tecnología didáctica de los profesores, especialmente al considerarlos de acuerdo con los ciclos donde ejercen la enseñanza, esto es, educadoras de párvulo, profesores de primer ciclo básico y profesores de segundo ciclo básico. En este último ciclo, en las escuelas del estudio predominan profesores de enseñanza media.

Así, como elemento fundamental en el discurso de las educadoras aparece la importancia del uso de material concreto y su relación con el desarrollo del pensamiento de los niños. En el discurso de profesores de primer ciclo, además de la importancia otorgada al material concreto, aparece la preocupación por el insuficiente tiempo para "enseñar" todos los contenidos del libro. Aparece aquí también la importancia de la resolución de problemas y el desarrollo de habilidades. La perspectiva de estos profesores es que los contenidos son medios para el aprendizaje y no un fin en sí mismo. No parecen sentirse autorizados como para modificar la secuencia de contenidos propuesta por el Método Singapur. Los profesores de segundo ciclo, por su parte, ponen a los contenidos matemáticos en el centro de su discurso, sobre dónde deberían/podrían ubicarse en la trayectoria curricular de básica, resguardando las articulaciones entre ciclos. El material concreto, de manera general, es aludido en un papel subsidiario. Muestran gran seguridad respecto de mover de lugar ciertos temas, buscando descomprimir a la vez que viabilizar la enseñanza en términos de tiempo. Por ejemplo expresan: "Hemos tenido que reorganizar los capítulos de los libros en función de la estructura y ejes de la matemática; si no, parece una mezcolanza". En el discurso se subordina la lógica del aprendizaje de los niños a la lógica de las propias matemáticas.

Finalmente, los profesores manifiestan dificultades, especialmente los de primer ciclo, para "soltar" el paradigma tradicional que busca instalar un único procedimiento, tal como expresan a continuación: "Entonces ese paso entre soltar la estrategia que les sirve, que ordena y clarifica el problema, a empezar a usar sus propias estrategias de niño, es un tema; y es algo en que uno debe ir con mucha cautela, permitiéndoles soltar la estrategia, sin que se 
122 QUÉ Y CUÁNTO APRENDEN DE MATEMÁTICAS LOS ESTUDIANTES DE BÁSICA CON EL MÉTODO SINGAPUR: EVALUACIÓN DE IMPACTO Y DE FACTORES INCIDENTES EN EL APRENDIZAJE, ENFATIZANDO EN LA BRECHA DE GÉNERO - L. Espinoza, C. Matus, J. Barbe, J. Fuentes y F. Márquez

queden sin nada. Ese intermedio resulta no ser muy fácil de lograr, y en ocasiones tenemos algunos problemas".

5.3.2. Estadios de apropiación del Método Singapur en las escuelas Esta parte del estudio partió por analizar, relacionar y triangular toda la información recogida de cada una de las escuelas, sus docentes y estudiantes, considerando el avance entre los años 2015 y 2016. Dichas escuelas llevan al menos seis años de implementación del método. Posteriormente, se realizó un contraste entre los resultados por escuela, con el interés de poder comprender y describir el proceso de apropiación del Método Singapur vivido por cada una de ellas en términos de una trayectoria que pasa por distintos "estadios". Con ello, se lograron determinar algunas características comunes en dicho proceso de apropiación, así como también algunas diferencias.

Esta reflexión y análisis permitió distinguir y precisar al menos tres estadios en dicho proceso. A continuación se describen las características esenciales de cada estadio, las que han sido identificadas utilizando las categorías de análisis de los procesos de estudio construidas en Gellert et al. (2013). 


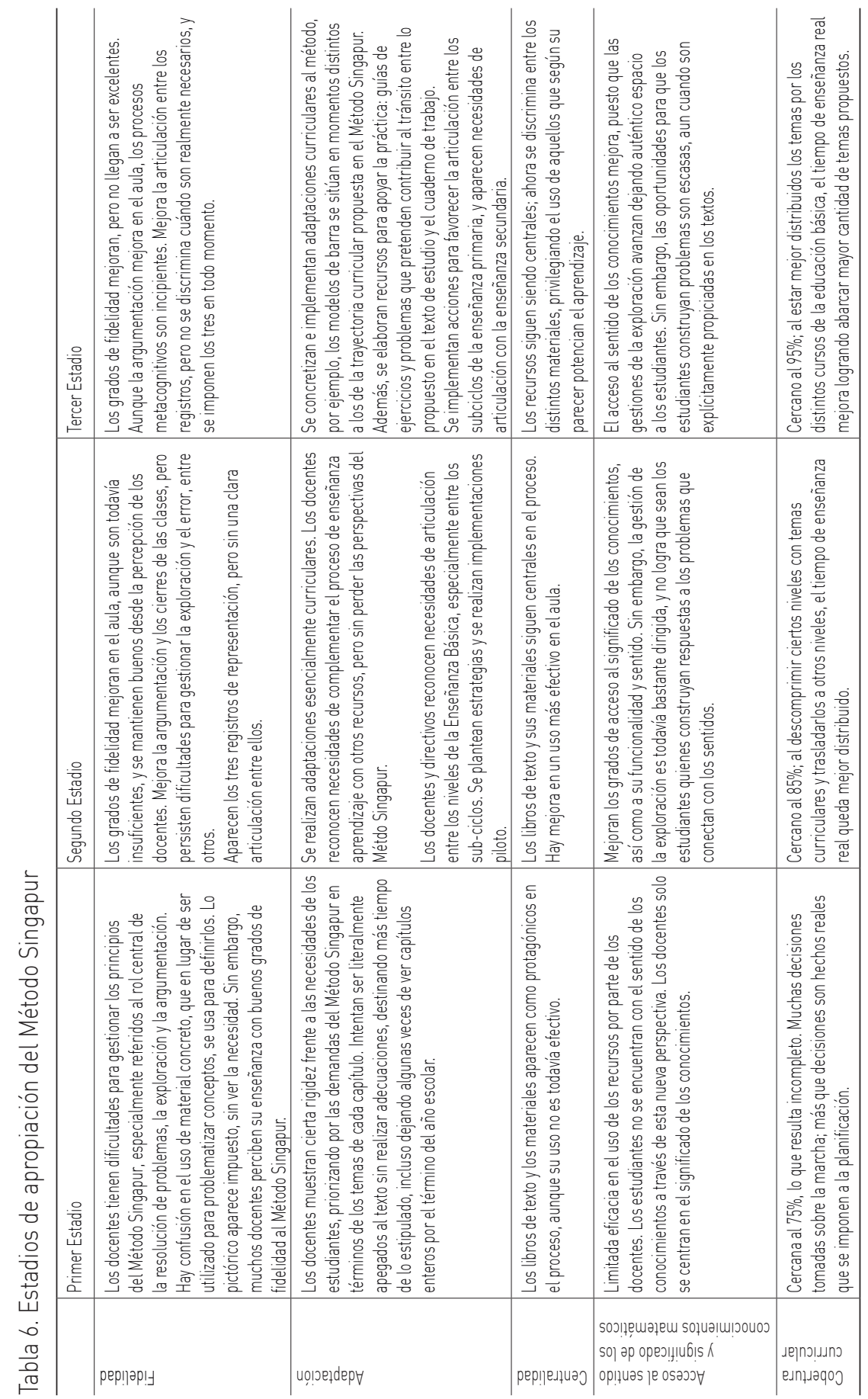


124 QUÉ Y CUÁNTO APRENDEN DE MATEMÁTICAS LOS ESTUDIANTES DE BÁSICA CON EL MÉTODO SINGAPUR: EVALUACIÓN DE IMPACTO Y DE FACTORES INCIDENTES EN EL APRENDIZAJE, ENFATIZANDO EN LA BRECHA DE GÉNERO - L. Espinoza, C. Matus, J. Barbe, J. Fuentes y F. Márquez

Dadas las grandes resistencias de los docentes frente a los cambios en la enseñanza, la "fidelidad" con la propuesta del Método Singapur es el criterio más complejo de alcanzar por la mayoría de docentes al realizar la enseñanza a lo largo del proceso. Por el contrario, el criterio de "centralidad" de los recursos para su uso efectivo en el aula es, de manera general, el criterio mejor logrado por estos docentes, mientras que el criterio de adaptación del método a las necesidades de sus estudiantes aparece con buenos niveles de logro, en parte importante de los docentes participantes.

Una vez descritos los estadios y analizando las distintas dimensiones y categorías por escuela, se encontró que una de las tres escuelas, pese a llevar seis años en el proceso de instalación del Método Singapur, está en el primer estadio de apropiación. La mayoría de sus docentes siguen presentando dificultades para gestionar clases acordes con los principios del Método Singapur y sus reflexiones son mayoritariamente descriptivas. Es importante señalar que en esta escuela hay un alto porcentaje de estudiantes con dificultades de aprendizaje, y también una rotación considerable de profesores. Otra escuela se podría situar en el segundo estadio; si bien sus docentes todavía tienen dificultades para gestionar los principios del Método Singapur en el aula, han experimentado grandes avances en algunos de ellos que son clave. Sus niveles de reflexión les permiten analizar el proceso de apropiación del método de tal modo de identificar falencias y fortalezas, aunque todavía con una visión parcelada sin comprender globalmente lo que como escuela les sucede y necesitan. La tercera escuela se podría situar en el tercer estadio de apropiación. Segura de sus logros como institución hasta antes de implementar el Método Singapur, tiene la confianza suficiente como para adaptarlo a sus propias estrategias y organización de la enseñanza, sin perder la esencia de la metodología. Los grados de reflexión pedagógica y didáctica de los docentes y directivos acerca del método son elevados, lo que les permite actuar con flexibilidad y consistencia. Dichos grados de reflexión, por su complejidad y diversidad, merecen ser estudiados con mayor profundidad, por lo que podrían ser objeto de estudio de otra investigación. 


\subsubsection{Condiciones institucionales y factores que favorecen y/o} dificultan la apropiación del Método Singapur

Estos resultados son encontrados a partir del análisis y triangulación de la información obtenida a partir de los grupos focales, registros de visitas y entrevistas.

Entre los factores que dificultan la apropiación a nivel institucional se encuentran los siguientes:

- Escasez de espacios para la reflexión pedagógica en las escuelas y para el trabajo en equipo.

- Alta rotación docente.

- Exigencias por alcanzar mejores resultados de aprendizaje en corto plazo. Por lo general, en los primeros años de implementación del Método Singapur, los resultados de aprendizaje en las pruebas, tanto internas como estandarizadas, tienden a ser irregulares.

- Insuficiente cantidad de material concreto.

- Interferencias planteadas por los padres. Por lo general, suelen presentarse diferencias entre la metodología que plantea el Método Singapur y la experiencia de aprendizaje que han tenido los padres. Por ello aparecen exigencias y tensiones por conseguir mayores explicaciones y justificaciones del método.

- Insuficiente cantidad de pruebas disponibles en los textos en relación con las demandas de calificaciones de las escuelas.

En relación con los factores que dificultan la apropiación a nivel curricular se cuentan:

- El tiempo para la implementación del Método Singapur en las escuelas, por lo general, es inferior al requerido por el propio método. Esto exige en muchos casos adaptaciones curriculares para descomprimir los contenidos tratados en un nivel educativo y trasladarlos hacia otros, en búsqueda de optimizar los tiempos y, a la vez, lograr cobertura curricular a lo largo de la educación básica.

- Algunas diferencias entre la progresión de los objetivos de aprendizaje de las bases curriculares y la progresión propuesta en los textos Pensar sin límites.

- Exigencias de adaptaciones curriculares en $4^{\circ}$ básico para responder a la prueba Simce. 
126 QUÉ Y CUÁNTO APRENDEN DE MATEMÁTICAS LOS ESTUDIANTES DE BÁSICA CON EL MÉTODO SINGAPUR: EVALUACIÓN DE IMPACTO Y DE FACTORES INCIDENTES EN EL APRENDIZAJE, ENFATIZANDO EN LA BRECHA DE GÉNERO - L. Espinoza, C. Matus, J. Barbe, J. Fuentes y F. Márquez

- Necesidad de construir recursos de aprendizaje que recubran espacios de trabajo de los estudiantes que, según los profesores, el Método Singapur no contempla. Por ejemplo, guías de ejercicios y problemas, pruebas, entre otras.

Por último, entre los factores que dificultan la apropiación a nivel del profesorado se destacan:

- El paradigma tradicional basado fuertemente en la explicación del docente es el dominante en estas escuelas.

- La formación matemática y didáctica de los docentes son insuficientes para abordar y encarnar los principios del Método Singapur en el aula. En particular esto se refiere a otorgar un rol protagónico a la resolución de problemas dentro del proceso de aprendizaje, abrir espacios y plantear oportunidades para la argumentación de los estudiantes, realizar una gestión de los errores en el aprendizaje que lo sitúe como una oportunidad y no como una carencia, etc.

- Las creencias en el cuerpo docente acerca de la matemática como un cuerpo rígido, estático y poco articulado de conceptos, que desconoce la funcionalidad y génesis de los conocimientos matemáticos.

Ahora bien, en relación con los factores que favorecen la apropiación del método se pueden mencionar los siguientes:

- Disposición positiva e innovadora de los equipos directivos, que los lleva a comprometer a toda la comunidad educativa en la implementación del método.

- Organización estructural de las prácticas pedagógicas que demuestra sensibilidad y comprensión del complejo fenómeno de aprendizaje y una favorable disposición hacia evaluar y ajustar las decisiones tomadas.

- El Método Singapur permite unificar y homogenizar las prácticas de la enseñanza; establece un referente común sobre el cual es posible observar las prácticas con los mismos lentes, discutir, reflexionar y actuar colectivamente.

- La alta motivación de los estudiantes por estudiar matemáticas con este nuevo método actúa como la principal razón que justifica el cambio de paradigma y los esfuerzos pedagógicos y didácticos 
asociados. "Dejó de ser tema la matemática en el colegio y pasó a ser una de las asignaturas más entretenidas del currículo".

- Necesidad de organización por equipos de docentes, que se ha ido incrementando a lo largo de la experiencia de apropiación del Método Singapur.

- Disponibilidad y acceso al material concreto y a los libros de texto.

- La capacitación de los docentes aparece como un factor clave y estrictamente necesario para que el Método Singapur sea apropiado e implantado eficazmente en el colegio.

- El rol protagónico del material concreto dentro del proceso de aprendizaje, cuidadosamente articulado con distintos registros de representación pictórica y simbólica.

- Toma de decisiones pedagógicas que van siendo cada vez más acertadas en el tiempo, por ejemplo, asignación criteriosa de docentes en los distintos niveles de enseñanza, apoyos específicos a docentes con mayores debilidades, etc.

- Padres comprometidos con el proceso de aprendizaje de sus hijos.

\section{Conclusiones}

La investigación planteó cinco preguntas orientadoras del estudio, relacionadas con: aspectos curriculares del Método Singapur; posible impacto en resultados de aprendizaje y mejoras en la brecha de género; características del proceso de apropiación del método por parte de docentes, estudiantes y sus escuelas; $y$ factores que favorecen y dificultan dicho proceso de apropiación.

Los análisis permitieron evidenciar, en primer lugar, que los textos Pensar sin límites poseen altos grados de cobertura, consistencia y coherencia con las bases curriculares. No obstante, una mirada superficial a ellos podría llevar a conclusiones erradas, puesto que varios temas son tratados de manera integrada a otros más amplios, no siendo visibles en primera instancia.

En segundo lugar, los resultados permitieron mostrar un impacto positivo en el logro de los aprendizajes y desarrollo de habilidades matemáticas en estudiantes de $4^{\circ}$ básico que utilizan el Método Singapur respecto de estudiantes del mismo nivel que utilizan 
128 QUÉ Y CUÁNTO APRENDEN DE MATEMÁTICAS LOS ESTUDIANTES DE BÁSICA CON EL MÉTODO SINGAPUR: EVALUACIÓN DE IMPACTO Y DE FACTORES INCIDENTES EN EL APRENDIZAJE, ENFATIZANDO EN LA BRECHA DE GÉNERO - L. Espinoza, C. Matus, J. Barbe, J. Fuentes y F. Márquez

otras metodologías de enseñanza de las matemáticas. Además se verificó una reducción estadísticamente significativa en la brecha de género de los estudiantes que vivieron la aplicación del MS.

En tercer lugar, los resultados han permitido describir el proceso de apropiación del Método Singapur vivido por las escuelas participantes en términos de una trayectoria que pasa por distintos estadios, en cada uno de los cuales las instituciones enfrentan diversos obstáculos, al tiempo que resguardan condiciones que favorecen el avance en dicha apropiación. En dos de las tres escuelas, sus profesores y directivos fueron adquiriendo a lo largo de este tránsito mayores herramientas para reflexionar y gestionar procesos de enseñanza acordes con los principios del Método Singapur. De esta manera, se constató una creciente riqueza y variedad en el uso de los recursos para el aprendizaje por parte de docentes y de estudiantes, así como mayores conocimientos didácticos usados en la práctica de aula. Claro está que, así como las escuelas presentaron aspectos comunes en esta trayectoria, también mostraron algunas diferencias específicas. En cualquier caso, se pudo constatar, en distintos grados, un progreso pedagógico y didáctico provocado por el proceso de implementación y apropiación del Método Singapur en estas escuelas. Dicho progreso se observó a nivel del profesorado, de sus estudiantes y también a nivel más macro de toma de decisiones de carácter institucional que estructuran las prácticas de la enseñanza en las escuelas.

Finalmente, se lograron identificar factores que favorecen la implementación y apropiación del Método Singapur en las escuelas, entre ellos la capacitación de los docentes, la capacidad de organización interna, la toma de decisiones asertiva y comprometida con los cambios requeridos, y el entusiasmo de los docentes por utilizar el método. Asimismo, se identificaron factores que dificultan dicho proceso de implementación y que actúan como obstáculos para su apropiación. Por ejemplo, grandes diferencias entre el paradigma tradicional dominante en la enseñanza de las matemáticas en estas escuelas y el paradigma en el cual se inscribe el Método Singapur, así como escasez de tiempos para la reflexión docente y el trabajo colectivo que les permita asumir el cambio en las prácticas de enseñanza. 


\section{Referencias}

Agencia de Calidad de la Educación, ACE. (2013). Diferencias actitudinales entre hombres y mujeres en matemática. Análisis de los resultados de la prueba PISA 2012. Recuperado de https://s3.amazonaws.com/archivos. agenciaeducacion.cl/documentos-web/Papers/2013_12_Diferencias_ actitudinales_entre_hombres_y_mujeres_en_matematica_resultados_ Prueba_PISA_2012.pdf

American Institutes for Research, AIR. (2005). What the United States can learn from Singapores world-class mathematics system: An exploratory study (and what Singapore can learn from the United States). Washington, DC: American Institutes for Research. Recuperado de: http://www.air.org/ sites/default/files/downloads/report/Singapore_Report_Bookmark_ Version1_0.pdf

Barbé, J., Bosch, M., Espinoza, L., \& Gascón, J. (2005). Didactic restrictions on the teacher's practice. The case of limits of functions in Spanish high schools. Educational Studies in Mathematics, 59(1-3), 235-268. http://dx.doi.org/10.1007/s10649-005-5889-z

Barquero, B., Bosch, M., \& Gascón, J. (2013). The ecological dimension in the teaching of mathematical modeling at university. Recherches en didactique des mathématiques, 33(3), 307-338.

Brousseau, G. (1990). Le contrat didactique: Le milieu, Recherches en Didactique des Mathématiques, 9(3), 308-336.

Brousseau, G. (1994). Concours externe de recrutement des professeurs d'Ecole. Annales 1993. Université de Bourdeaux: Publicaciones LADIST.

Brousseau, G. (1997). Theory of didactical situations in mathematics. Didactique des mathématiques, 1970-1990 (N. Balacheff, R. Sutherland, \& V. Warfield Trans. \& Eds.). Dordrecht: Kluwer Academic Publishers.

Chevallard, Y. (1985). La transposición didáctica. Del saber sabio al saber enseñado. Buenos Aires: Aique.

Chevallard, Y. (1997). Familière et problématique, la figure du professeur. Recherches en Didactique des Mathématiques, 17(3), 17-54.

Chevallard, Y. (1999). L'analyse des pratiques enseignantes en théorie anthropologique du didactique. Recherches en Didactiques des Mathématiques, 19(2), 22-266.

Chevallard, Y. (2012). Teaching mathematics in tomorrow's society: A case for an oncoming counter paradigm. En Sung Je Cho (Ed.), The proceedings of the 12th International Congress on Mathematical Education (pp. 173-187). Springer open. https://dx.doi.org/10.1007/978-3-31912688-3_13 
130 QUÉ Y CUÁNTO APRENDEN DE MATEMÁTICAS LOS ESTUDIANTES DE BÁSICA CON EL MÉTODO SINGAPUR: EVALUACIÓN DE IMPACTO Y DE FACTORES INCIDENTES EN EL APRENDIZAJE, ENFATIZANDO EN LA BRECHA DE GÉNERO - L. Espinoza, C. Matus, J. Barbe, J. Fuentes y F. Márquez

Centro de Estudios, Mineduc. (2013). Serie evidencias: El rol de la evaluación de programas en las políticas públicas: el caso del proyecto piloto "Textos de Singapur". Recuperado de: http://centroestudios.mineduc.cl/ tp_enlaces/portales/tp5996f8b7cm96/uploadImg/File/Evidencias/ A2_N24_Textos_Singapur.pdf

Cochran-Smith, M. \& Fries, K. (2005). The AERA Panel on Research and Teacher Education: Context and Goals. En M. Cochran-Smith \& K. Zeichner (Eds.), Studying teacher education. The Report of the AERA panel on research and teacher education (pp. 37-68). New Jersey: Lawrence Erlbaum Associates.

Conner, J. M. (2010). A study of the Singapore math program math in focus, state test results. Educational research: Institute of America. Recuperado de http://www.hmhelearning.com/math/mathinfocus/mif12/resources/ MiF NJ Ask State Test Score Analysis.pdf

Espinoza, L., Barbé, J., y Gálvez, G. (2009). Estudio de fenómenos didácticos vinculados a la enseñanza de la aritmética en la educación básica chilena. Enseñanza de las ciencias, 27(2), 157-168.

Espinoza, L., Barbé, J., y Gálvez, G. (2011). Limitaciones en el desarrollo de la actividad matemática en la escuela básica: el caso de la aritmética escolar. Estudios Pedagógicos, 37(1), 105-125. http://dx.doi. org/10.4067/s0718-07052011000100006

Gellert, U., Barbé, J., \& Espinoza, L. (2013). Towards a local integration of theories: Codes and praxeologies in the case of computer-based instruction. Educational Studies in Mathematics, 82(2), 303-321. http:// dx.doi.org/10.1007/s10649-012-9427-5

Gertler, P., Martínez, S., Premand, P., Rawlings, L., y Vermeersch, C. (2011). La evaluación de impacto en la práctica. Washington, DC: Banco Internacional de reconstrucción y fomento/Banco Mundial.

McKinsey \& Company. (2007). Cómo hicieron los sistemas educativos con mejor desempeño del mundo para alcanzar sus objetivos. Recuperado de http:// www.oei.es/pdfs/documento_preal41.pdf

Ministerio de Educación de Singapur. (2012). Primary mathematics teaching and learning syllabus. Singapore: Ministry of Education.

Mullis, I. V. S., Martin, M. O., Foy, P., \& Arora, A. (2012). TIMSS 2011 International results in mathematics. Chestnut Hill, MA: TIMSS \& PIRLS International Study Center, Boston College.

Organización para la Cooperación y el Desarrollo Económicos, OCDE. (2009). Los docentes son importantes: atraer, formar y conservar a los docentes eficientes. Recuperado de http://www.waece.org/enciclopedia/2/ Los\%20docentes\%20son\%20importantes.pdf 
Organización para la Cooperación y el Desarrollo Económicos, OCDE. (2014). PISA 2012 Results: What students know and can do (Volume I, Revised edition, February 2014): Student performance in mathematics, reading and science. Paris: OCDE Publishing

Porlán, R., Rivero, A, y Martín del Pozo, R. (1998). Conocimiento profesional y epistemología de los profesores II: estudios empíricos y conclusiones. Enseñanza de las Ciencias, 16(2), 271-288.

Organización de las Naciones Unidas para la Educación, la Ciencia y la Cultura, Unesco. (2012). Atlas mundial de la igualdad de género en la educación. Paris: Autor.

Recibido: 01/09/2016

Aceptado: 26/11/2016 